Pacific Journal of Mathematics

GDY OF ABSOUTTE EXTESSOR S 


\title{
A STUDY OF ABSOLUTE EXTENSOR SPACES
}

\author{
CARLOS R. Borges
}

In the enclosed paper, we will prove, among others, the following results: (a) A sufficient condition that a space $L$ be an absolute extensor for the class of stratifiable spaces in that $L$ be hyperconnected (this is a refinement of the concept of equiconnected space). This condition is also necessary if $L$ is a metrizable space. (b) Every hypogeodesic space is hyperconnected. (c) Every equiconnected space is $\infty$-hyperconnected. (d) Every $\infty$-hyperconnected space is an absolute extensor for the class of $C W$-complexes of Whitehead.

Some of the merits of our results are the following: (1) The well-known extension theorem of Dugundji (Theorem 4.1 of [2]) as well as part of Theorem 4.3 of [1] are immediate consequences of our Theorem 4.1. (2) It is easily verified that the space $Y$ of Theorem 3.4 of [3] (of course, we need to interchange the roles of the first and second variables of $F$ in order to agree with Dugundji's definition of $\lambda$-stable), is locally hypogeodesic and, therefore, Theorem 3.4 of [3] is an easy consequence of our Theorems 3.3 and 4.4. (3) Our results generalize Theorems 3 and 4 of Himmelberg [4] and Theorem 3.4 of Dugundji [3] by removing the stringent hypothesis that the range space be metrizable.

Our many attempts to solve the question "Is every equiconnected metrizable space an AE (metrizable) ${ }^{1 "}$, which is raised in [3], have, so far, ended in failure. However, our Theorem 4.3 offers a partial solution which leads us to conjecture an affirmative answer to this question, especially in view of the "replacement-by-polytopes" technique of Dugundji [2] (If only we could do it!).

2. Definitions. Throughout, let $P_{n-1}$ denote the unit simplex in Euclidean $n$-space $R^{n}$ (i.e., $P_{n-1}=\left\{t \in R^{n} \mid \sum_{i=1}^{n} t_{i}=1\right.$ and each $\left.t_{i} \geqq 0\right\}$, $I$ the closed unit interval, and $A^{n}$ the $n$-fold cartesian product of any set $A$. Furthermore, let $\delta_{i}: A^{n} \rightarrow A^{n-1}$ be the map defined by

$$
\delta_{i}\left(a_{1}, \cdots, a_{n}\right)=\left(a_{1}, \cdots, a_{i-1}, a_{i+1}, \cdots, a_{n}\right)
$$

for $i=1,2, \cdots, n$.

It seems appropriate to start our definitions by recollecting the concept of an "equiconnected space", which was introduced by Fox [4] and is called a $U C$-space by Serre [9, p. 490], not only because

${ }^{1}$ We use the following abbreviations: $\mathrm{AE} \equiv$ absolute extensor, $\mathrm{ANE} \equiv$ absolute neighborhood extensor, $\mathrm{AR} \equiv$ absolute retract, $\mathrm{ANR} \equiv$ absolute neighborhood retract. 
it is closely related to the new concepts we will introduce but also because it will make them more plausible. It should also be observed that the similarity of our Definitions 2.2 and 2.3 with Definitions 1.1 and 5.1 of Michael [8] is, by far, not accidental.

Definition 2.1. A space $L$ is equiconnected if there exists a continuous map $F: L \times L \times I \rightarrow L$ such that $F(a, b, 0)=a, F(a, b, 1)=b$ and $F(a, a, t)=a$ for all $(a, b) \in L \times L$ and $t \in I$. (The function $F$ will be called an equiconnecting function.) The space $L$ is said to be locally equiconnected if $F$ is defined only on $U \times I$ with $U$ some neighborhood of the diagonal of $L \times L$.

Definition 2.2. A space $L$ will be called respectively hyperconnected, $m$-hyperconnected, if there exists functions $h_{i}: L^{i} \times P_{i-1} \rightarrow L$, for $i=1,2, \cdots$, which satisfy conditions (a), (b), (c) and conditions (a), (b), (d) respectively:

(a) $t \in P_{n-1}$ and $t_{i}=0$ implies $h_{n}(x, t)=h_{n-1}\left(\delta_{i} x, \delta_{i} t\right)$ for each $x \in L^{n}$ and $n=2,3, \cdots$,

(b) for each $x \in L^{n}$, the map $t \rightarrow h_{n}(x, t)$, from $P_{n-1}$ to $L$, is continuous,

(c) for each $x \in L$ and neighborhood $U$ of $x$, there exists a neighborhood $V$ of $x$ such that

$$
\bigcup_{i=1}^{\infty} h_{i}\left(V^{i} \times P_{i-1}\right) \subset U
$$

and $V \subset U$,

(d) for each $x \in L$ and neighborhood $U$ of $x$, there exists a neighborhood $V$ of $x$ such that

$$
\bigcup_{i=1}^{m} h_{i}\left(V^{i} \times P_{i-1}\right) \subset U
$$

and $V \subset U$ (we should observe that, in this case, the functions $h_{k}$, for $k \geqq m+1$, may be assumed to be constant functions). The space $L$ will be called $\infty$-hyperconnected provided that $L$ is $m$-hyperconnected for $m=1,2, \cdots$. The space $L$ will be called locally hyperconnected provided that, for each $x \in X$, there exists a neighborhood of $x$ which is hyperconnected. We similarly define locally m-hyperconnected and locally o-hyperconnected.

Definition 2.3. A space $L$ is said to be hypogeodesic if there exists a function $F: L \times L \times I \rightarrow L$ satisfying the following conditions:

(a) for $x, y \in L$ and $t \in I, F(x, y, 0)=x, F(x, y, 1)=y$ and $F(x, x, t)=x$,

(b) for each $y \in L$, the map $(x, t) \rightarrow F(x, y, t)$, from $L \times I$ to $L$, is continuous, 
(c) for each $x, a, y \in L$ and neighborhood $U$ of $y$, there exist neighborhoods $V$ of $a$ and $W$ of $y$ ( $V$ and $W$ depend on $x$ ) with $W \subset U$, such that

$$
F(a, x, t) \in W \quad \text { implies } F(b, x, t) \in U
$$

for any $b \in V$,

(d) for each $x \in L$ there exist neighborhood bases $\left\{V_{\alpha}\right\}$ and $\left\{U_{\alpha}\right\}$ of $x$, with $V_{\alpha} \subset U_{\alpha}$ for each $\alpha$, such that

$$
y \in V_{\alpha}, z \in U_{\alpha} \text { implies } F(z, y, t) \in U_{\alpha}
$$

for each $t \in I$.

(The function $F$ will be called an hypogeodesic function for $L$.)

The space $L$ is said to be locally hypogeodesic if $F$ is defined only on $U \times I$ with $U$ some neighborhood of the diagonal on $L \times L$.

Clearly, every locally convex linear topological space is hypogeodesic, and every linear topological space is equiconnected. Furthermore, every equiconnecting function is easily seen to satisfy conditions (a), (b), and (c) of Definition 2.3.

3. Hypo, equi and hyper.

THEOREM 3.1. If $L$ is hypogeodesic then $L$ is hyperconnected.

Proof. (Similar to the proof of Proposition 5.3 of Michael [8].) Throughout this proof we will make use of the following notation:

(1) If $x \in L^{n+1}$, then $\hat{x} \in L^{n}$ is defined by $\widehat{x}_{i}=x_{i}$ for $i=1, \cdots, n$.

(2) If $t \in P_{n}$ and $t_{n+1} \neq 1$ then $\hat{t} \in P_{n-1}$ is defined by

$$
\hat{t}_{i}=\frac{t_{i}}{1-t_{n+1}} \text { for } i=1, \cdots, n \text {. }
$$

Clearly $\delta_{i} \hat{x}=\left(\widehat{\left.\delta_{i} x\right)}\right.$ and $\delta_{i} \hat{t}=\widehat{\left(\delta_{i} t\right)}$, whenever $t \in P_{n-1}, x \in L^{n}$ and $1<i<n$.

Let $h_{1}: L \times\{1\} \rightarrow L$ be defined by $h_{1}(x, 1)=x$, and let $F$ be an hypogeodesic function for $L$. By induction, assume we have defined maps $h_{i}: L^{i} \times P_{i-1} \rightarrow L$, for $i=1, \cdots, n$, which satisfy parts (a) and (b) of Definition 2.2. Now let

$$
h_{n+1}(x, t)= \begin{cases}x_{n+1} & \text { if } t_{n+1}=1 \\ F\left(h_{n}(\hat{x}, \hat{t}), x_{n+1}, t_{n+1}\right) & \text { if } t_{n+1} \neq 1 .\end{cases}
$$

Let us check that the functions $h_{n}$ satisfy conditions (a), (b) and (c) of Definition 2.2.

2.2(a). Clearly $h_{2}(x, t)=h_{1}\left(\delta_{i} x, \hat{o}_{i} t\right)$ whenever $t_{i}=0$. Hence let us 
assume that $h_{n}(x, t)=h_{n-1}\left(\delta_{i} x, \delta_{i} t\right)$ whenever $t_{i}=0$ and let us shown that $h_{n+1}(x, t)=h_{n}\left(\delta_{i} x, \delta_{i} t\right)$ whenever $t_{i}=0(1 \leqq i \leqq n+1)$ : If $i=n+1$ then $h_{n+1}(x, t)=F\left(h_{n}\left(\widehat{x}, \delta_{n+1} t\right), x_{n+1}, 0\right)=h_{n}\left(\widehat{x}, \delta_{n+1}|t|\right)=h_{n}\left(\delta_{n+1} x, \delta_{n+1} t\right)$ by (3) and Definition 2.3(a). If $i<n+1$ and $t_{n+1}=1$, then $h_{n+1}(x, t)=$ $x_{n+1}=h_{n}\left(\delta_{i} x, \delta_{i} t\right)$, by (3). If $i<n+1$ and $t_{n+1} \neq 1$, then

$$
\begin{aligned}
h_{n+1}(x, t) & =F\left(h_{n}(\hat{x}, \hat{t}), x_{n+1}, t_{n+1}\right) \\
& =F\left(h_{n-1}\left(\hat{\delta}_{i} \widehat{x}, \delta_{i} \hat{t}\right), x_{n+1}, t_{n+1}\right) \\
& =F\left(h_{n-1}\left(\widehat{(}\left(\widehat{\delta_{i} x}\right),\left(\widehat{\delta_{i} t}\right)\right), x_{n+1}, t_{n+1}\right)=h_{n}\left(\delta_{i} x, \delta_{i} t\right) .
\end{aligned}
$$

By induction, we get that the functions $h_{n}$ satisfy Definition 2.2(a).

2.2(b). Proof by induction. Clearly the map $t \rightarrow h_{1}(x, t)=x$, from $P_{0}$ to $L$, is continuous. Suppose that the map $t \rightarrow h_{n}(w, t)$, from $P_{n-1}$ to $X$, is continuous, for each $w \in L^{n}$. Pick a fixed point $x \in L^{n+1}$ and let us show that the map $t \rightarrow h_{n+1}(x, t)$, from $P_{n}$ to $L$, is also continuous: Let $s \in P_{n}$. Then

Case 1. $s_{n+1} \neq 1$. For some neighborhood $U$ of $s$ in $P_{n}, t \in U$ implies $t_{n+1} \neq 1$. Therefore, for $t \in U, h_{n+1}(x, t)=F\left(h_{n}(\hat{x}, \hat{t}), x_{n+1}, t_{n+1}\right)$ and hence the continuity of the map $t \rightarrow h_{n+1}(x, t)$ at $s \in P_{n}$, follows from Definition $2.3(\mathrm{~b})$ and the inductive hypothesis. (Indeed, pick sequence $t(1), t(2), \cdots$ in $U \subset P_{n}$ such that $\lim _{i} t(i)=s$. Then one easily sees that $\lim _{i} \widehat{t(i)}=\widehat{s}$ and thus $\lim _{i} h_{n}(\widehat{x}, \widehat{t}(\hat{i}))=h_{n}(\widehat{x}, \widehat{s})$ by the inductive hypothesis. Consequently, $\lim _{i} h_{n+1}\left(x,(t(i))=\lim _{i} F\left(h_{n}(\hat{x}, \widehat{t}(\hat{i}))\right.\right.$, $x_{n \div 1},\left(t(i)_{n+1}\right)=F\left(h_{n}(\hat{x}, \hat{s}), x_{n+1}, s_{n+1}\right)=h_{n+1}(x, s)$ which shows that the map $t \rightarrow h_{n+1}(x, t)$ is continuous at $s$.)

Case 2. $s_{n+1}=1$. Then $h_{n+1}(x, s)=x_{n+1}$, by (3). We will show that $h_{n+1}(x, t)$ is close to $x_{n+1}$ if $t_{n+1}$ is close to 1 , as follows: Let $A=\left\{h_{n}(\widehat{x}, t) \mid t \in P_{n-1}\right\}$ and, for each $\tau \in I$, let $f_{:}: A \rightarrow X$ be defined by

$$
f_{\ulcorner}(\alpha)=F\left(a, x_{n+1}, \tau\right) \text {. }
$$

Then

$$
\begin{aligned}
& h_{n+1}(x, t)=f_{t_{n+1}}\left(a_{t}\right) \quad \text { for some } a_{t} \in A \text { if } t_{n+1} \neq t, \\
& h_{n+1}(x, t)=x_{n+1} \quad \text { if } t_{n+1}=1 .
\end{aligned}
$$

Therefore, in order to prove that the map $t \rightarrow h_{n+1}(x, t)$ is continuous at $s$, it suffices to show that $f_{\tau} \rightarrow f_{1}$ uniformly as $\tau \rightarrow 1$ (note that $f_{1}(a)=x_{n+1}$ for each $a \in A$, by Definition 2.3(a)): From Definition 2.3(b) we get that $f_{\tau} \rightarrow f_{1}$ pointwise. Moreover $A$ is compact, because $h_{n}$ is continuous and $\{x\} \times P_{n}$ is compact, and the family $\left\{f_{\tau} \mid \tau \in I\right\}$ is evenly continuous (see page 235 of [7]), by Definition 2.3(c). (Let $a \in A \subset L$ and $y \in L$ and let $U$ be a neighborhood of $y$. By Definition 2.3(c), pick neighborhoods $V$ of $a$ and $W$ of $y$ ( $V$ and $W$ depend on $x_{n+1}$ ) with 
$W \subset U$ such that whenever $F\left(a, x_{n+1}, t\right) \in W$ then $F\left(b, x_{n+1}, t\right) \in U$ for all $b \in V$. Then we obviously get that $f_{\tau}(V) \subset U$ whenever $f_{\tau}(a) \in W$, which shows that the family $\left\{f_{\tau} \mid \tau \in I\right\}$ is evenly continuous.) By Theorem 23 (page 237) of [7], the family $\left\{f_{\tau} \mid \tau \in I\right\}$ is equicontinuous (note that, for each $a \in A,\left\{f_{\tau}(a) \mid \tau \in I\right\} \subset F\left(A \times\left\{x_{n+1}\right\} \times I\right)$ a compact subset of $L$, because of Definition 2.3(b)). Therefore, the topologies of pointwise convergence and uniform convergence coincide on $\left\{f_{\tau} \mid \tau \in I\right\}$, by Theorem 15 (page 232) of [7], and hence $f_{\tau} \rightarrow f_{1}$ uniformly as $\tau \rightarrow 1$.

It is now easily seen that the map $t \rightarrow h_{n+1}(x, t)$ is continuous at each $s \in P_{n}$.

2.2(c). Let $x \in L$ and open $U \subset L$ such that $x \in U$. Then, by Definition 2.3(d), there exists neighborhoods $V$ and $W$ of $x$ such that $V \subset W \subset U$ and $F(z, y, t) \in W$ whenever $y \in V$ and $z \in W$. By an inductive argument, one easily sees that

$$
\bigcup_{n=1}^{\infty} h_{n}\left(V^{n} \times P_{n-1}\right) \subset W \subset U,
$$

which completes the proof.

THEOREM 3.2. If $L$ is equiconnected then $L$ is $\infty$-hyperconnected.

Proof. Exactly the same as the proof of Theorem 3.1, except that we must verify that the functions $h_{n}, n=1,2, \cdots$, satisfy condition (d) of Definition 2.2: Let us first observe that, for each neighborhood $U$ of $x$ one easily finds a neighborhood $V$ of $x$ with $V \subset U$,

$$
F(V \times V \times I) \subset U,
$$

because $F$ is continuous, $F(x, x, t)=x$ for each $t \in I$ and $I$ is compact. Clearly $L$ is 1 -hyperconnected. Therefore let us assume that $L$ is $n$ hyperconnected and show that $L$ is $(n+1)$-hyperconnected. Pick $x \in L$ and neighborhood $U$ of $x$. Then pick neighborhoods $V$ and $W$ of $x$ such that

$$
F(V \times V \times I) \subset U, h_{n}\left(W^{n} \times P_{n-1}\right) \subset V, W \subset V \subset U .
$$

Using the definition of $h_{n+1}$, it is trivial to check that

$$
h_{n+1}\left(W^{n+1} \times P_{n}\right) \subset U \text {. }
$$

This concludes our inductive argument and completes the proof.

Because of the proofs of Theorems 3.1 and 3.2, the following result is clearly valid and easily verified: 
THEOREM 3.3. If $L$ is locally hypogeodesic (locally equiconnected) then $L$ is locally hyperconnected (locally $\infty$-hyperconnected).

\section{Extension theorems.}

THEOREM 4.1. Every hyperconnected space $L$ is an $\mathrm{AE}$ (stratifiable).

Proof. The proof of this result is very similar to the proof of Theorem 4.3 in [1]. We will thus indicate the general procedure without details. We briefly comment on some recurring notation throughout ensuing proof: Letting $U \rightarrow\left\{U_{n}\right\}_{n=1}^{\infty}$ be a stratification of $X$ (see footnote 1), we let $n(U, x)=\min \left\{n \mid x \in U_{n}\right\}$ and $U_{x}=U_{n(U, x)}-(X-\{x\})_{n(U, x)}^{-}$, for each open $U \subset X$ and $x \in U$. It is easily seen (see Lemma 4.1 of [1]) that each $U_{x}$ is an open neighborhood of $x, U_{x} \cap V_{y} \neq \varnothing$ implies that $x \in V$ or $y \in U$ (indeed, $n(U, x) \leqq n(V, y)$ implies that $y \in U$ ).

Now let $X$ be a stratifiable space, $A$ a closed subset of $X, f: A \rightarrow L$ a continuous function. Let $W=X-A, W^{\prime}=\left\{x \in W \mid x \in U_{y}\right.$ for some $y \in A$ and open $U$ containing $y\}$ and $m(x)=\max \{n(U, y) \mid y \in A$ and $x \in U_{y}$, for each $x \in W^{\prime}$. It is easily seen that $m(x)<n(W, x)<\infty$.

Using the paracompactness of $W$, let $\mathscr{V}$ be an open locally finite (with respect to $W$ ) refinement of $\left\{W_{x} \mid x \in W\right\}$. For each $V \in \mathscr{V}$ pick $x_{V} \in W$ with $V \subset W_{x_{V}}$. If $x_{V} \in W^{\prime}$ pick $a_{V} \in A$ and open $S_{V}$ containing $a_{V}$ such that $x_{V} \in\left(S_{V}\right)_{a_{V}}$ and $n\left(S_{V}, a_{V}\right)=m\left(x_{V}\right)$; if $x_{V} \in W$, let $a_{V}$ be the fixed point $a_{0} \in A$.

Let $\left\{p_{V} \mid V \in \mathscr{Y}\right\}$ be a partition of unity subordinated to $\mathscr{V}$; and define $g: X \rightarrow L$ by

$$
\begin{gathered}
g(x)=f(x) \quad \text { for } x \in A \\
g(x)=h_{n}\left(\left(f\left(a_{V_{1}}\right), \cdots, f\left(a_{V_{n}}\right)\right),\left(p_{V_{1}}(x), \cdots, p_{V_{n}}(x)\right) \quad \text { for } x \in W,\right.
\end{gathered}
$$

where $V_{1}, \cdots, V_{n}$ are the only elements $V \in \mathscr{V}$ such that $p_{V}(x) \neq 0$, for $x \in X-A$. Clearly $g$ is a well defined function from $X$ to $L$. It is not quite obvious that $g$ is continuous anywhere, as was the case in Theorem 4.3 of [1].

We will first show that $g$ is continuous at each point $b \in A$. Let 0 be any open subset of $L$ containing $f(b)$ and let $H$ be an open subset of 0 such that $f(b) \in H$ and $\bigcup_{n=1}^{\infty} h_{n}\left(H^{n} \times P_{n-1}\right) \subset 0$. Since $f$ is continuous there exists an open neighborhood $N$ of $b$ such that $f(A \cap N) \subset$ $H \subset 0$. It is easily seen that $g\left(\left(N_{b}\right)_{b}\right) \subset 0$, which shows that $g$ is continuous at each $b \in A$.

Finally we show that $g$ is continuous at each $x \in X \rightarrow A$. Let 0 be a neighborhood of

$$
g(x)=h_{m}\left(\left(f\left(a_{V_{1}}\right), \cdots, f\left(a_{V_{m}}\right)\right),\left(p_{V_{1}}(x), \cdots, p_{V_{m}}(x)\right)\right)
$$


and let $N$ be a neighborhood of $x$ which intersects only finitely many $V \in \mathscr{Y}$; say $V_{1}, \cdots, V_{m}, \cdots, V_{m+k}$. By Definition 2.2(b), there exists a neighborhood $W$ of $\left(p_{V_{1}}(x), \cdots, p_{V_{m}}(x), 0, \cdots, 0\right) \in P_{m+k-1}$ which is mapped into 0 by the continuous map

$$
t \rightarrow h_{m+k}\left(\left(f\left(a_{V_{1}}\right), \cdots, f\left(a_{r_{m+n}}\right), t\right)\right. \text {. }
$$

Define a map $p: N \rightarrow P_{m+k-1} \subset I^{m+k}$ by $p(y)=\left(p_{V_{1}}(y), \cdots, p_{V_{m+k}}(y)\right)$ for each $y \in N$. Since $p$ is clearly continuous, then there exists a neighborhood $U$ of $x$ such that $p(U) \subset W$. It is now easily seen that $g(N \cap U) \subset 0$, with the help of Definition 2.2(a), which shows that $g$ is continuous at each $x \in X-A$, thus completing the proof.

It is easily seen that the preceding proof remains valid if we assume that $L$ is only $(n+1)$-hyperconnected and $X-A$ is $n$-dimensional (in the covering sense), for then we can choose the open cover $\mathscr{q}$ to be of order $n$ and thus define the function $g$ in terms of $f$ and $h_{n+1}$ only. We have, therefore, proved the following result.

Theorem 4.2. Let $X$ be a stratifiable space, $A$ a closed subset of $X, L$ an $(n+1)$-hyperconnected space and $f: A \rightarrow L$ a continuous function. If $\operatorname{dim}(X-A) \leqq n$ then $f$ has a continuous extension $g$ : $X \rightarrow L$.

The following result, when combined with Theorem 3.2, provides a partial answer to the following question which is raised in [3]: Is every equiconnected matrizable space an $\mathrm{AE}$ (metrizable)?

THEOREM 4.3. Every c-hyperconnected space $L$ is an $\mathrm{AE}(C W-$ complex of Whitehead).

Proof. Let $K$ be a $C W$-complex, $A$ a closed subset of $K$ and $f$ : $A \rightarrow L$ a continuous function. For each $n$, let $K_{n}$ be the $n$-skeleton of $K$. It is well-known that $\operatorname{dim} K_{n}$ is finite for each $n$. Therefore, by theorem 4.2 (note that $K$ is stratifiable because of Theorem 7.2 of [1], for example) and induction, we can find continuous functions

$$
g_{n}: A \cup K_{n} \rightarrow L \quad \text { for } n=1,2, \cdots
$$

such that

$$
g_{n} \mid A=f \text { and } g_{n+1} \mid\left(A \cup K_{n}\right)=g_{n}
$$

for each $n$. Now define $g: K \rightarrow L$ by

$$
g(x)=g_{n}(x) \quad \text { if } x \in A \cup K_{n} .
$$

It is easily seen that $g$ is a well-defined continuous extension of $f$ to 
all of $X$, which completes the proof.

It is easily seen that all the preceding results of this section have local analogues. For the sake of completeness, let us state and prove one.

THEOREM 4.4. Every locally hyperconnected space $L$ is an ANE (stratifiable).

Proof. By Theorem 4.1, $L$ is a local AE (stratifiable) (i.e., for each $a \in L$ there exists a neighborhood $N$ of a such that $N$ is an $\mathrm{AE}$ (stratifiable)). Consequently, by Theorem 19.2 of Hanner [5], $L$ is an ANE (stratifiable).

\section{Characterization of $\mathrm{AR}$ (metrizable) spaces.}

THEOREM 5.1. A metrizable space $M$ is an AE (stratifiable) if and only if $M$ is hyperconnected.

Proof. Since the "if" part is an immediate consequence of Theorem 4.1, we direct our attention to the "only if" part (the basic technique is extracted from Michael [8]): Embed $M$ in a Banach space $B$ and let $H$ be the closed convex hull of $M$ in $B$. Then there exists a retraction $r: H \rightarrow M$. For each $n$, define $h_{n}: M^{n} \times P_{n} \rightarrow M$ by

$$
h_{n}(z, t)=r\left(\sum_{i=1}^{n} t_{i} z_{i}\right), \quad \text { for }(z, t) \in M^{n} \times P_{n} .
$$

Clearly, each $h_{n}$ satisfies conditions (a) and (b) of Definition 2.2. Since $r$ is continuous, for each $x \in M$ and open subset $U$ of $H$ with $x \in U$, there exists a convex open set $V \subset H$ with $x \in V \subset U$ such that

$$
r(V \cap M) \subset r(V) \subset U \cap M \text {; }
$$

hence $\left.\bigcup_{i=1}^{\infty} h_{i}(V \cap M)^{i} \times P_{i-1}\right) \subset U \cap M$, and hence the functions $h_{n}$ satisfy condition (c) of Definition 2.2. Consequently $M$ is hyperconnected.

\section{BIBLIOGRAPHY}

1. C. J. R. Borges, On stratifiable spaces, Pacific J. Math. 17 (1966), 1-16.

2. J. Dugundji, An extension of Tietze's theorem, Pacific J. Math. 1 (1951), 353-367.

3 . L Locally equiconnected spaces and absolute neighborhood retracts, Fund. Math. 62 (1965), 187-193.

4. R. H. Fox, On fiber spaces, II, Bull. Amer. Math. Soc. 49 (1943), 733-735.

5. O. Hanner, Retraction and extension of mappings of metric and nonmetric spaces, Ark. Mat. 2 (1952), 315-360.

6. C. J. Himmelberg, Some theorems on equiconnected aud locally equiconnected spaces, Trans, Amer. Math. Soc. 115 (1965), 43-53. 
7. J. L. Kelley, General topology, Van Nostrand, New York, 1955.

8. E. A. Michael, Convex structures and continuous selections, Canad. J. Math. 11 (1959), 556-575.

9. J. P. Serre, Homlogie singulière des espaces fibrés, Ann. of Math. 54 (1951), 425-505.

Received August 15, 1968, and in revised form October 17, 1968. This research was supported by University of California Faculty Fellowship.

University of CALIFornia, DAVIS 



\title{
PACIFIC JOURNAL OF MATHEMATICS
}

\author{
EDITORS
}

\author{
H. ROYDEN \\ Stanford University \\ Stanford, California \\ RICHARD PIERCE \\ University of Washington \\ Seattle, Washington 98105
}

\author{
J. DUGUNDJI \\ Department of Mathematics \\ University of Southern California \\ Los Angeles, California 90007 \\ BASIL GORDON \\ University of California \\ Los Angeles, California 90024
}

\section{ASSOCIATE EDITORS}

E. F. BECKENBACH
B. H. NeumanN

F. WOLF
K. YoshidA

\section{SUPPORTING INSTITUTIONS}

\author{
UNIVERSITY OF BRITISH COLUMBIA \\ CALIFORNIA INSTITUTE OF TECHNOLOGY \\ UNIVERSITY OF CALIFORNIA \\ MONTANA STATE UNIVERSITY \\ UNIVERSITY OF NEVADA \\ NEW MEXICO STATE UNIVERSITY \\ OREGON STATE UNIVERSITY \\ UNIVERSITY OF OREGON \\ OSAKA UNIVERSITY \\ UNIVERSITY OF SOUTHERN CALIFORNIA
}

\author{
STANFORD UNIVERSITY \\ UNIVERSITY OF TOKYO \\ UNIVERSITY OF UTAH \\ WASHINGTON STATE UNIVERSITY \\ UNIVERSITY OF WASHINGTON

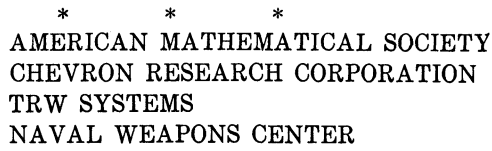

The Supporting Institutions listed above contribute to the cost of publication of this Journal, but they are not owners or publishers and have no responsibility for its content or policies.

Mathematical papers intended for publication in the Pacific Journal of Mathematics should be in typed form or offset-reproduced, double spaced with large margins. Underline Greek letters in red, German in green, and script in blue. The first paragraph or two must be capable of being used separately as a synopsis of the entire paper. It should not contain references to the bibliography. Manuscripts, in duplicate if possible, may be sent to any one of the four editors. Please classify according to the scheme of Math. Rev. 36, 1539-1546. All other communications to the editors should be addressed to the managing editor, Richard Arens, University of California, Los Angeles, California, 90024.

50 reprints are provided free for each article; additional copies may be obtained at cost in multiples of 50 .

The Pacific Journal of Mathematics is published monthly. Effective with Volume 16 the price per volume (3 numbers) is $\$ 8.00$; single issues, $\$ 3.00$. Special price for current issues to individual faculty members of supporting institutions and to individual members of the American Mathematical Society: $\$ 4.00$ per volume; single issues $\$ 1.50$. Back numbers are available.

Subscriptions, orders for back numbers, and changes of address should be sent to Pacific Journal of Mathematics, 103 Highland Boulevard, Berkeley, California, 94708.

PUBLISHED BY PACIFIC JOURNAL OF MATHEMATICS, A NON-PROFIT CORPORATION

Printed at Kokusai Bunken Insatsusha (International Academic Printing Co., Ltd.), 7-17, Fujimi 2-chome, Chiyoda-ku, Tokyo, Japan. 


\section{Pacific Journal of Mathematics}

\section{Vol. 31, No. $3 \quad$ BadMonth, 1969}

George E. Andrews, On a calculus of partition functions .................. 555

Silvio Aurora, A representation theorem for certain connected rings ............ 563

Lawrence Wasson Baggett, A note on groups with finite dual spaces ............. 569

Steven Barry Bank, On majorants for solutions of algebraic differential equations in regions of the complex plane ............................... 573

Klaus R. Bichteler, Locally compact topologies on a group and the corresponding continuous irreducible representations ......................... 583

Mario Borelli, Affine complements of divisors ....................... 595

Carlos Jorge Do Rego Borges, A study of absolute extensor spaces ............. 609

Bruce Langworthy Chalmers, Subspace kernels and minimum problems in Hilbert

spaces with kernel function ...................................... 619

John Dauns, Representation of L-groups and F-rings................. 629

Spencer Ernest Dickson and Kent Ralph Fuller, Algebras for which every

indecomposable right module is invariant in its injective envelope ...........

Robert Fraser and Sam Bernard Nadler, Jr., Sequences of contractive maps and fixed

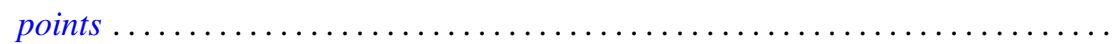

Judith Lee Gersting, A rate of growth criterion for universality of regressive

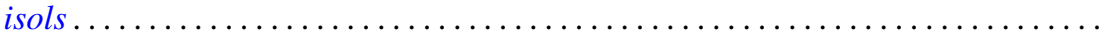

Robert Fred Gordon, Rings in which minimal left ideals are projective ............

Fred Gross, Entire functions of several variables with algebraic derivatives at certain algebraic points

W. Charles (Wilbur) Holland Jr. and Stephen H. McCleary, Wreath products of ordered permutation groups .........................

W. J. Kim, The Schwarzian derivative and multivalence .................. 717

Robert Hamor La Grange, Jr., On $(\mathrm{m}-\mathrm{n})$ products of Boolean algebras ......... 725

Charles D. Masiello, The average of a gauge ........................ 733

Stephen H. McCleary, The closed prime subgroups of certain ordered permutation

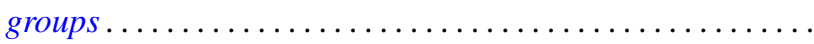

Richard Roy Miller, Gleason parts and Choquet boundary points in convolution measure algebras ...............................

Harold L. Peterson, Jr., On dyadic subspaces ........................ 773

Derek J. S. Robinson, Groups which are minimal with respect to normality being intransitive........................................... 777

Ralph Edwin Showalter, Partial differential equations of Sobolev-Galpern type . . . 787

David Slepian, The content of some extreme simplexes ................... 795

Joseph L. Taylor, Noncommutative convolution measure algebras ............. 809

B. S. Yadav, Contractions of functions and their Fourier series ............... 827

Lindsay Nathan Childs and Frank Rimi DeMeyer, Correction to automorphisms of separable algebras" ....................... 833

Moses Glasner and Richard Emanuel Katz, Correction to: "Function-theoretic degeneracy criteria for Riemannian manifolds".............

Satish Shirali, Correction to: "On the Jordan structure of complex Banach

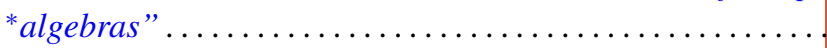

